\title{
Estudo das Propriedades Reológicas, Morfológicas e Mecânicas de Blendas Injetadas de Polipropileno com Poliamidas Reforçadas com Fibras de Vidro.
}

\author{
G. Villoutreix, A Haddout, N.R. Demarquette, J. Villoutreix e J. D. Acétarin
}

\begin{abstract}
Resumo: Neste trabalho foi estudada a influência da adição de compatibilizantes, da composição e dos parâmetros de processamento nas propriedades reológicas, morfológicas e mecânicas de blendas de polipropileno com poliamidas aromáticas, adicionadas de fibras de vidro, obtidas por moldagem por injeção. Os resultados obtidos mostram que a adição de um compatibilizante melhora a adesão entre as fases da blenda, apresentando um papel emulsificante e estabilizador da morfologia da blenda. Utilizando-se um reômetro "in line" na máquina de injeção, e através de observações morfológicas, foi mostrado que ocorre uma evolução significativa da morfologia em função dos parâmetros de processamento e da composição da blenda. A orientação das fibras de vidro em função da posição dentro do molde foi também estudada neste trabalho, demonstrando a existência de três camadas distintas no molde.
\end{abstract}

Palavras-chave: Blendas poliméricas, moldagem por injeção, reologia, polipropileno, poliamidas, compatibilização.

\section{Introdução}

Blendas poliméricas representam hoje em dia cerca de $30 \%$ do mercado de polímeros ${ }^{[1,2]}$ e são atualmente um dos setores de maior crescimento na indústria de polímeros. Taxas de crescimento de mercado de mais de $17 \%$ têm sido publicadas nos últimos 30 anos nos Estados Unidos, o que representa um crescimento até 5 vezes maior do que de outros setores da indústria. Cada ano são mais de 4.500 pedidos de patentes para novas blendas poliméricas e não menos de 20.000 trabalhos científicos sobre blendas são publicados ${ }^{[2]}$.
O sucesso das blendas poliméricas é devido especialmente a um custo menor de desenvolvimento (quando comparado ao custo de desenvolvimento de novas moléculas), à possibilidade das blendas combinarem propriedades dos diferentes componentes poliméricos (resultando em um material que pode ter grande resistência mecânica por exemplo), a um processamento mais fácil quando comparado ao processo de produtos multicamadas e a uma alternativa para reciclagem . Todas essas características fazem as misturas de polímeros materiais extremamente interessantes para numerosas aplicações, tais como na indústria automobilística e na indústria de embalagens.

G. Villoutreix, J. Villoutreix, J.D. Acétarin, Conservatoire National des Arts et Métiers Paris, France, A. Haddout, Département Génie Mécanique de l'Ecole Supérieure d'Electricité et de Mécanique, Casablanca, Marocco,N. R. Demarquette, Departamento de Engenharia Metalúrgica e de Materiais da Escola Politécnica da Universidade de São Paulo, SP. 
As propriedades de uma blenda polimérica são diretamente ligadas à qualidade da sua morfologia, que por sua vez é função de: a) características reológicas dos componentes que formam a blenda, b) compatibilidade entre os polímeros que formam a blenda, c) condições de processamento e d) composição da blenda.

Neste trabalho foram estudados os efeitos da compatibilização, da composição da blenda e dos parâmetros de processamento no comportamento reológico, e nas propriedades morfológicas e mecânicas de blendas de polipropileno com poliamidas com fibras de vidro. A orientação das fibras de vidro foi também estudada em função da posição dentro do molde de injeção.

Após a descrição dos materiais usados, os procedimentos experimentais para avaliar as propriedades reológicas e caracterizar a morfologia e as propriedades mecânicas das blendas são descritos. Para avaliar o comportamento reológico dos polímeros diretamente na máquina de injeção foi utilizado um reômetro "in line" acoplado a uma máquina injetora, desenvolvido nos laboratórios do Conservatoire National des Arts et Métiers, Paris, França ${ }^{[3,4]}$. A orientação das fibras e das fases da blenda no molde de injeção foi estudada utilizando-se um molde especial ${ }^{[5]}$. Neste artigo estão incluídas a apresentação e discussão dos resultados experimentais.

\section{Materiais e Métodos}

\section{Materiais}

Blendas de polipropileno (PP) com poliarilamida (PA) reforçadas com fibras de vidro foram estudadas neste trabalho. A poliarilamida é uma poliamida semiaromática obtida por policondensação de ácido adipamínico e de diamina metaxylilena ${ }^{[6]}$. A poliarilamida e o polipropileno são imísciveis em todas as concentrações e temperaturas ${ }^{[7]}$. Algumas blendas compatibilizadas com ácido maleico foram estudadas neste trabalho. Quando uma poliolefina é misturada com poliamida é possível enxertar ácido maleico na espinha dorsal da poliolefina, afim de compatibilizar os dois polímeros. Acredita-se que uma reação química entre o ácido maleico e a amina acontece durante a compatibilizaçãa ${ }^{[8]}$.

A Tabela 1 apresenta os materiais usados neste estudo; as blendas compatibilizadas foram fornecidas pela Solvay já compatibilizadas.

\section{Métodos Experimentais}

\section{Processamento}

As blendas, reforçadas ou não, foram injetadas numa máquina injetora Stork de 130 toneladas de força de fechamento e diâmetro de rosca de $52 \mathrm{~mm}$. A Tabela 2 mostra as condições de injeção usadas para injeção das blendas.

As amostras foram injetadas numa cavidade retangular com dimensões de $98 \mathrm{~mm}$ por $180 \mathrm{~mm}$ e uma espessura de $3,5 \mathrm{~mm}$ (bem inferior às outras dimensões de cavidade). A cavidade do molde utilizado é mostrada na Figura 1. As locações A, B, C, D correspondem às micrografias na Figura 9.

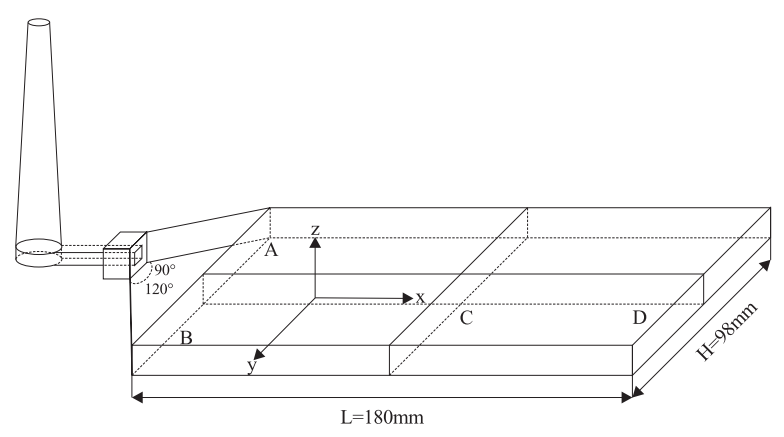

Figura 1. Molde de injeção

Tabela 1. Materiais utilizados neste estudo

\begin{tabular}{lcccc}
\hline \multicolumn{1}{c}{ Material } & $(\mathbf{P P})$ & $\left.\mathbf{( P P}_{\mathbf{f}}\right)$ & $\mathbf{P P} / \mathbf{P A}$ & $\mathbf{P P g} / \mathbf{P A}$ \\
\hline $\begin{array}{l}\text { Composição (PP/PA) } \\
(100 / 0)\end{array}$ & $\begin{array}{l}(100 / 0) \\
(30 / 70)\end{array}$ & $\begin{array}{c}(30 / 70) \\
(50 / 50) \\
(70 / 30)\end{array}$ \\
$\begin{array}{l}\text { Teor de fibras (\%) } \\
\begin{array}{l}\text { Blenda } \\
\text { compatibilizada }\end{array}\end{array}$ & não & não & não & sim \\
$\begin{array}{l}\text { Fornecedor } \\
\text { Elf }\end{array}$ & $\begin{array}{c}\text { Elf } \\
\text { Atochem }\end{array}$ & Atochem & Solvay & Solvay \\
\hline
\end{tabular}

\section{Comportamento reológico}

O comportamento reológico dos polímeros foi medido em um reômetro capilar, montado em uma máquina universal Inströn, equipada com uma matriz de L/D de 40 e com um diâmetro de barril de 9,5 $\mathrm{mm}$ para as taxas de cisalhamento de 10 até $10^{3} \mathrm{~s}^{-1}$. As correções de Rabinowitsch-Mooney foram utilizadas na obtenção dos dados reométricos.

O comportamento reológico para as taxas de cisalhamento de $10^{3} \mathrm{~s}^{-1}$ até $10^{7} \mathrm{~s}^{-1}$ foi medido utilizando-se um reômetro "in-line" acoplado a uma máquina 
Tabela 2. Parâmetros de injeção das blendas

\begin{tabular}{lcc}
\hline \multicolumn{1}{c}{ Parâmetros } & Unidades & Valores \\
\hline Velocidade de Injeção & $\mathrm{mm} / \mathrm{s}$ & $18,3-55,2$ \\
Temperatura de Injeção & ${ }^{\circ} \mathrm{C}$ & $250-280$ \\
Temperatura do Molde & ${ }^{\circ} \mathrm{C}$ & $60-120$ \\
Pressão de Injeção & $\mathrm{MPa}$ & 50 \\
Pressão de Recalque & $\mathrm{MPa}$ & 0,5 \\
Diâmetro da Rosca & $\mathrm{mm}$ & 52 \\
\hline
\end{tabular}

injetora. O reômetro "in-line" é incorporado na máquina injetora entre a bucha de injeção e a entrada no molde, como pode ser visto na Figura 2. O reômetro consiste em um cilindro de 2 a 4 mm de diâmetro, com 40 a 120 mm de comprimento. Os sensores de pressão e de temperatura são localizados em contato com o polímero fundido, planos com a parte interna do cilindro como pode ser visto na Figura 3. A informação adquirida pelos sensores é transmitida a um microcomputador para a obtenção dos resultados numéricos das pressões, temperaturas, taxas de cisalhamento, tensão de cisalhamento, e viscosidade dentro do cilindro.

A viscosidade aparente pode ser calculada utilizando-se a equação:

$$
\eta=\frac{\Delta \mathrm{PR}_{\mathrm{f}}^{4}}{2 \mathrm{LVR}_{\mathrm{V}}^{2}}\left[3+\frac{\mathrm{d}\left(\log \left(4 \mathrm{VR}_{\mathrm{V}}^{2} / \mathrm{R}_{\mathrm{f}}^{3}\right)\right)}{\mathrm{d}\left(\log \tau_{\mathrm{w}}\right)}\right]^{-1}
$$

onde $\eta$ é a viscosidade aparente, $\Delta \mathrm{P}$ é a perda de pressão dentro da matriz, $\mathrm{R}_{\mathrm{f}}$ é o raio da matriz, $\mathrm{L}$ o comprimento da matriz, $\mathrm{V}$ a velocidade de injeção, $\mathrm{R}_{\mathrm{v}}$ é o raio da rosca e $\tau_{\mathrm{w}}$ é a tensão de cisalhamento na parede.

Três sensores de pressão localizados ao longo da fieira permitem a medição de pressão de saída $\mathrm{P}_{\mathrm{s}} \mathrm{a}$ partir da qual a primeira diferença de tensões normais, $\mathrm{N}_{1}$, pode ser calculada de acordo $\operatorname{com}^{[4]}$ :

$$
\mathrm{N}_{1}=\mathrm{P}_{\mathrm{s}}\left[1+\frac{\mathrm{d}\left(\ln \mathrm{P}_{\mathrm{s}}\right)}{2 \mathrm{~d}\left(\ln \tau_{\mathrm{w}}\right)}\right]
$$

onde $\mathrm{P}_{\mathrm{s}}$ é a pressão de saída.

Utilizando este aparelho é possível conhecer o comportamento reológico de um polímero em condições reais de processamento para taxas de cisalhamento até $10^{7} \mathrm{~s}^{-1}$. Essas taxas de cisalhamento não podem ser obtidas normalmente com reômetros convencionais de laboratório.

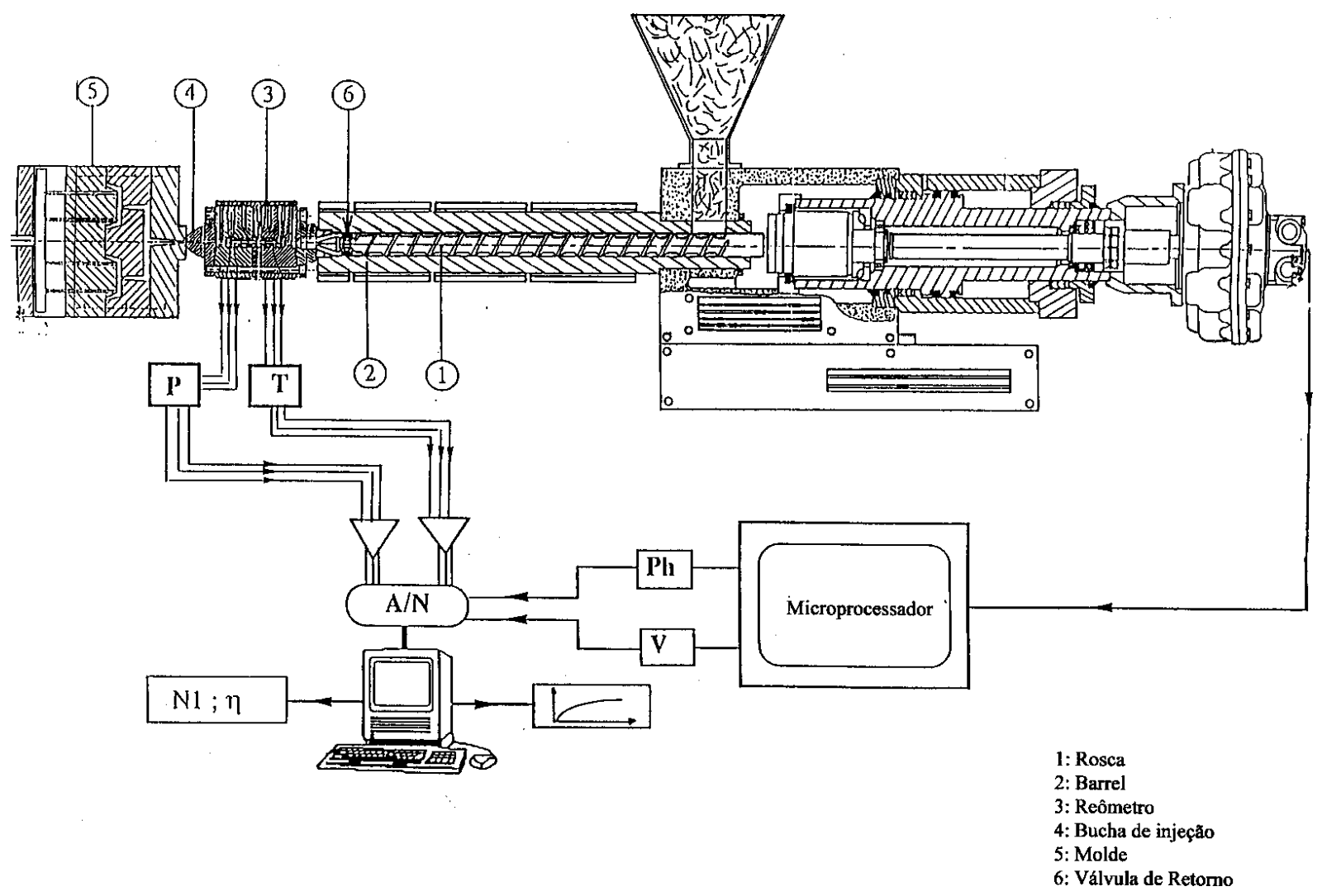

Figura 2. Reômetro "in line" montado na injetora 


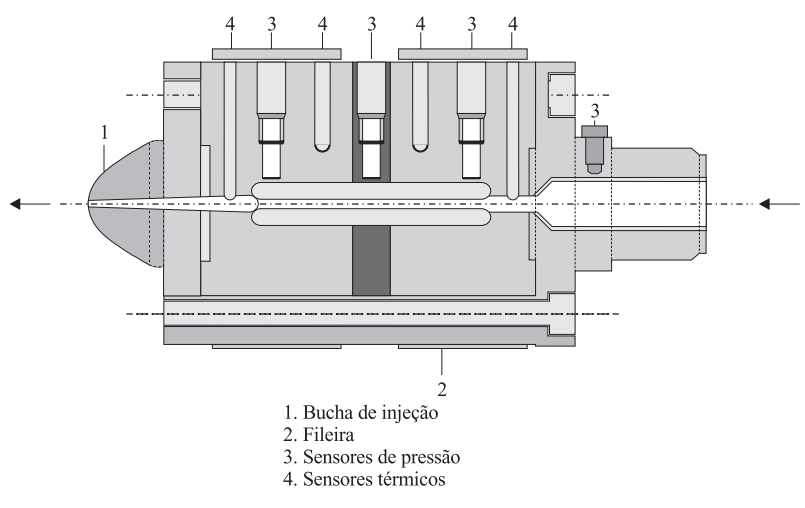

Figura 3. Celula do reômetro "in line"

\section{Estudo do comportamento morfológico}

As amostras de blendas de PP/PA foram cerradas e depois cortadas criogenicamente a uma temperatura de $77 \mathrm{~K}$. A fase dispersa (PP) foi dissolvida usando xileno a uma temperatura de $35^{\circ} \mathrm{C}$. As amostras foram então observadas no microscópio eletrônico de varredura.

A orientação das fibras nas amostras de PP/PA foi estudada por microscopia de reflexão. As superfícies das amostras foram polidas e atacadas quimicamente com ácido hidrofluorico para realçar a posição das fibras.

\section{Estudo do Comportamento mecânico da blenda}

As propriedades de impacto Charpy e de tração foram medidas para as blendas de acordo com normas ISO. Os corpos de prova foram injetados diretamente no formato apropriado para ensaios mecânicos.

\section{Resultados e Discussão}

\section{Comportamento reológico}

A Figura 4 mostra a viscosidade do polipropileno para duas temperaturas em função da taxa de cisalhamento. As medidas para taxas de cisalhamento baixa (10 até $10^{3} \mathrm{~s}^{-1}$ ) foram obtidas com o reômetro capilar e as medidas para taxas de cisalhamento acima de $10^{3} \mathrm{~s}^{-1}$ foram obtidas com o reômetro "in line" acoplado à máquina injetora. Pode ser observada a boa superposição dos dados obtidos com ambos reômetros mostrando a confiabilidade do reômetro "in line". Para taxas de cisalhamento até $10^{6} \mathrm{~s}^{-1} \mathrm{o}$ polipropileno mostra um comportamen-

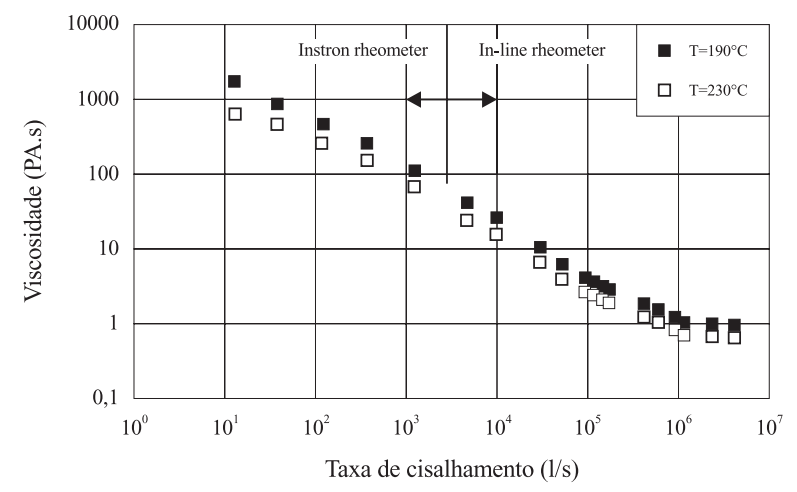

Figura 4. Viscosidade do PP (Instron e “in-line”)

to pseudoplástico. Para taxas de cisalhamento acima de $10^{6} \mathrm{~s}^{-1}$ ele mostra um comportamento newtoniano. Este tipo de comportamento nunca foi observado devido à impossibilidade dos reômetros convencionais de laboratório alcançarem taxas de cisalhamento elevadas.

A Figura 5 mostra o comportamento reológico do polipropileno reforçado com fibras para diferentes teores de fibras. Pode ser observado que a viscosidade é mais elevada para teores de fibras maiores em taxas de cisalhamento abaixo de $10^{4} \mathrm{~s}^{-1}$. Acima destas taxas de cisalhamento este comportamento não é mais observado; a viscosidade torna-se independente do teor de fibras de vidro. Este comportamento pode ser explicado pela orientação forçada das fibras com taxas de cisalhamento elevadas. Em outro trabalho ${ }^{[9]}$ foi estudada a orientação das fibras na saída da bucha de injeção para várias velocidades de injeção. Foi observado que para baixas velocidades de injeção as fibras orientam-se aleatoriamente gerando assim uma grande resistência ao escoamento. Para velocidades de injeção mais altas e consequentemente taxas de cisalhamento mais altas foi observado que as fibras se orientam na direção do fluxo e se localizam no centro do extrudado.

A Figura 6 mostra a viscosidade da blenda compatibilizada de polipropileno com poliaramida em

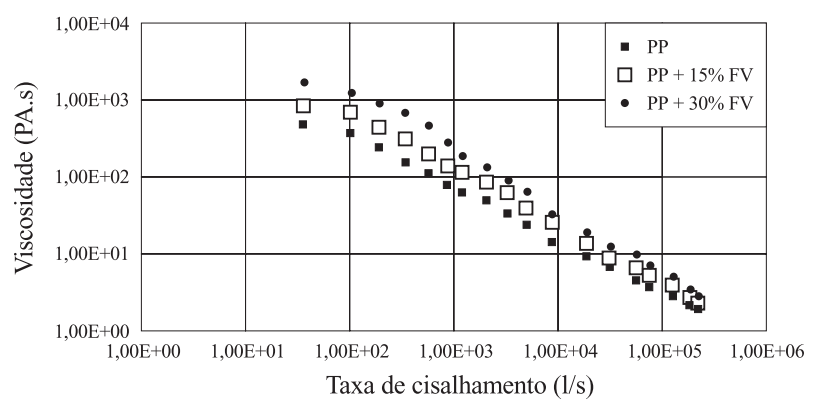

Figura 5. Comportamento reológico do PP para diferentes teores de fibras 


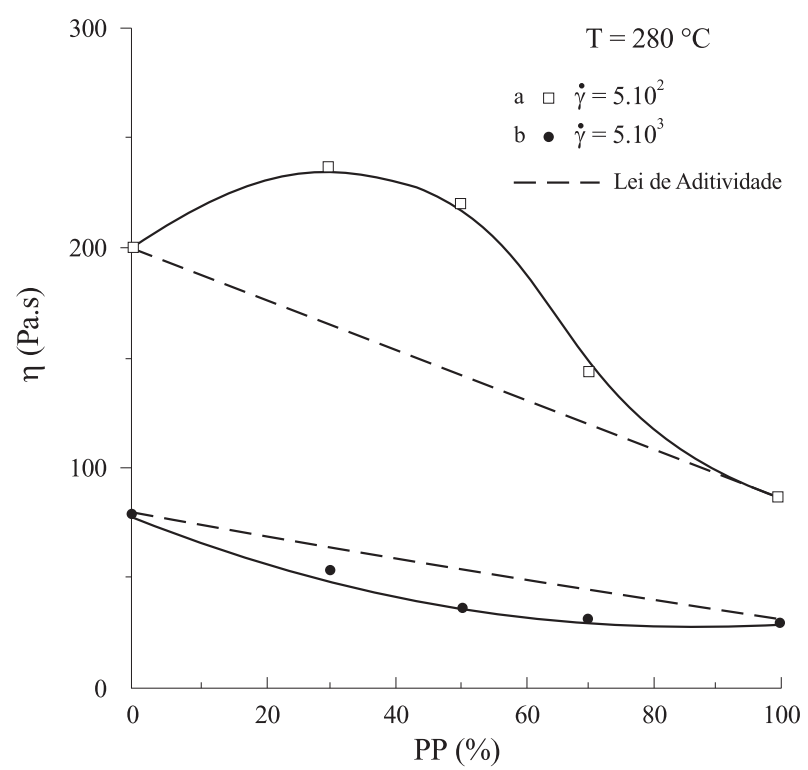

Figura 6. Comportamento reológico de blendas de $\mathrm{PP}_{\mathrm{g}} / \mathrm{PA}$

função da concentração de polipropileno para duas taxas de cisalhamento; os resultados para taxa de cisalhamento de $500 \mathrm{~s}^{-1}$ foram obtidos com o reômetro capilar e os resultados para taxa de cisalhamento de $5.10^{3} \mathrm{~s}^{-1}$ foram obtidos com o reômetro "in line" montado na máquina de injeção durante o processamento. Pode ser observado que para as duas taxas de cisalhamento estudadas, a blenda não segue a lei de aditividade clássica dada por ${ }^{[9]}$ :

$\ln \eta=\sum \mathrm{W}_{\mathrm{i}} \ln \eta_{\mathrm{i}}$

onde $\eta$ é a viscosidade da blenda, $\mathrm{W}_{\mathrm{i}}$ é a fração dos polímeros constituindo a blenda, e $\eta_{\mathrm{i}}$ é a viscosidade dos constituintes da blenda

Para taxas de cisalhamento de $500 \mathrm{~s}^{-1}$ a blenda mostra um desvio positivo e para taxas de cisalhamento de $5.10^{3} \mathrm{~s}^{-1}$ a blenda mostra um desvio negativo. Para blendas não miscíveis e não compatibilizadas, desvios negativos são sistematicamente observados para todas as taxas de cisalhamento. Quando as taxas de cisalhamento aumentam, os desvios são progressivamente mais negativos devido ao comportamento pseudoplástico dos polímeros e ao deslizamento entre as fases formadoras da blenda. Para blendas não miscíveis compatibilizadas, a compatibilização induz a uma interação entre as fases que se traduzem em um desvio positivo. Neste caso este desvio positivo é observado para pequenas taxas de cisalhamento (abaixo de $500 \mathrm{~s}^{-1}$ ). Quando as taxas de cisalhamento aumentam é observado um desvio negativo que pode ser atribuído a uma

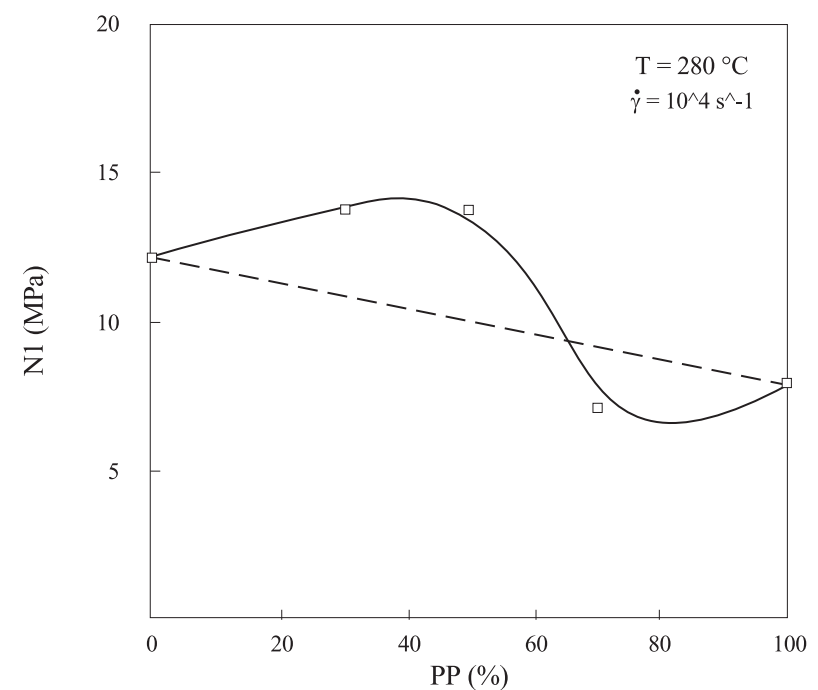

Figura 7. Evolução da primeira diferença de tensões normais em função da concentração de PP

demixagem da blenda para essas altas taxas de cisalhamento. A Figura 7 mostra a evolução da primeira diferença de tensões normais em função da concentração de polipropileno para uma taxa de cisalhamento de $10^{4} \mathrm{~s}^{-1}$. A primeira diferença de tensões normais mostra um desvio negativo-positivo da lei de mistura. O ponto de encontro entre os dados experimentais em função da composição com a reta correspondente à lei de mistura poderia corresponder a uma inversão de fase que aconteceria para altas taxas de cisalhamento para blendas com compatibilização incompleta. Com o reômetro "in line" apresentado aqui, foi possível detectar no processo de injeção se este fenômeno acontece.

\section{Estudo Morfológico}

\section{Orientação das fibras}

A Figura 8 mostra a orientação das fibras em função da espessura da camada na cavidade do molde para a blenda PP/PA não compatibilizada. Foram observadas 3 camadas na amostra moldada de $3.5 \mathrm{~mm}$ como pode ser visto na Figura 8. Na primeira camada (de pele), diretamente em contato com o molde, as fibras são orientadas randomicamente. $\mathrm{Na}$ segunda camada, as fibras são paralelas à direção do fluxo dentro do molde. Na terceira camada da região central (core), as fibras são perpendiculares ao fluxo. Este comportamento é devido às tensões de cisalhamento mais altas perto das paredes do molde durante o escoamento dos polímeros, e ao resfriamento mais rápido do polímero localizado 


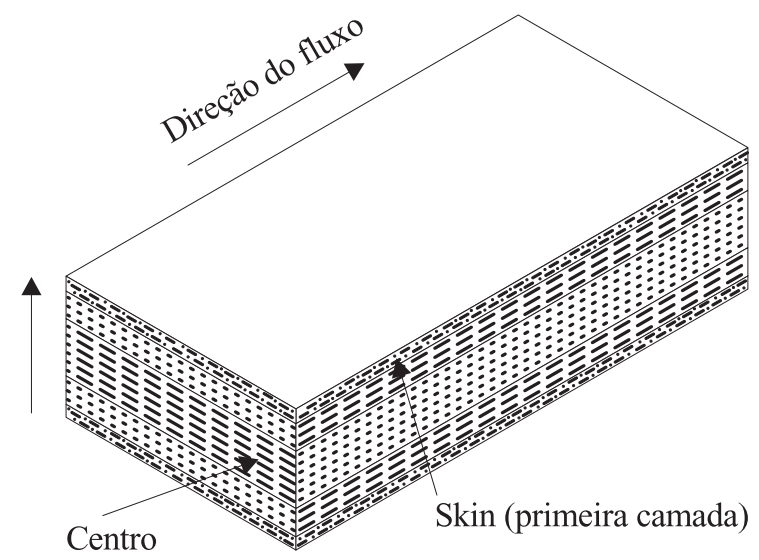

Figura 8. Orientação das fibras em função da espessura da camada na cavidade do molde.

perto das paredes. As tensões de cisalhamento altas orientam as fibras e o resfriamento mais rápido, perto das paredes, congela as morfologias. Observações similares já foram reportadas para polímeros amorfos, semi-cristalinos e blendas ${ }^{[10-18]}$.

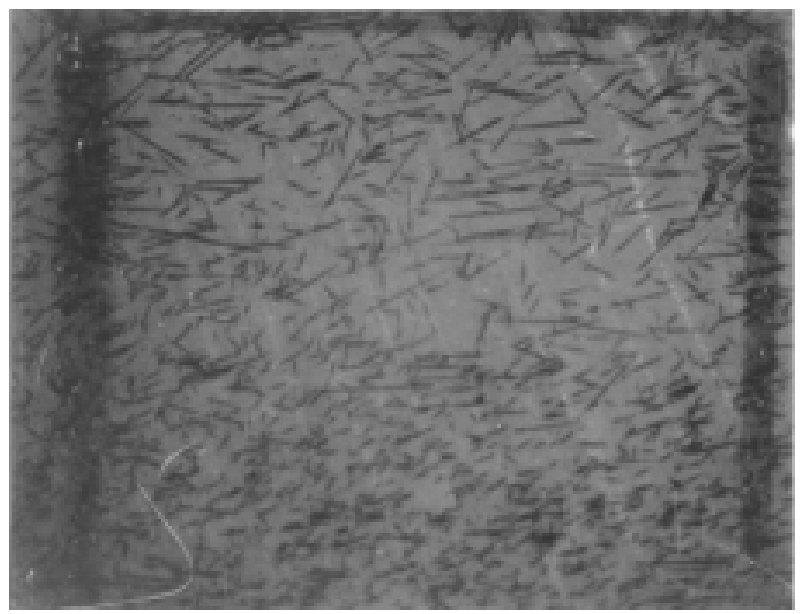

(a)

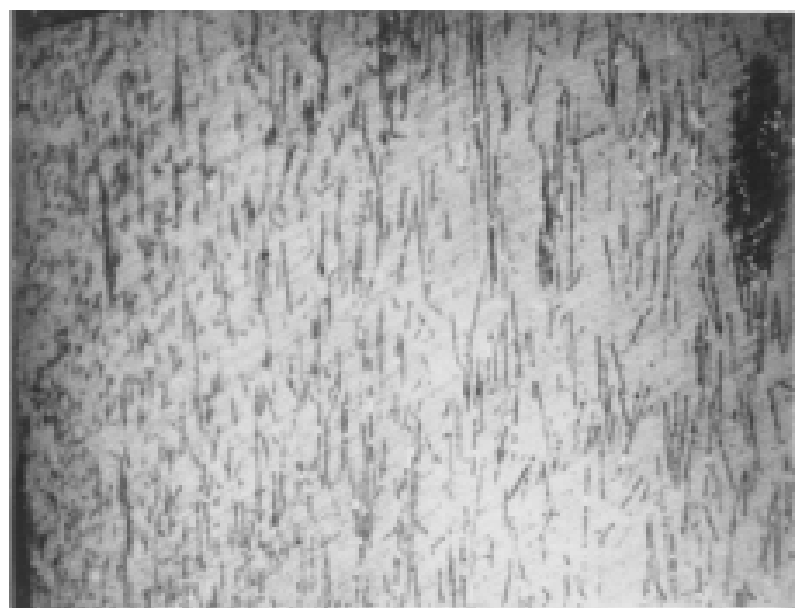

(c)

Figura 9. Orientação das fibras em função da posição dentro do molde
A orientação das fibras em função da posição do molde (direção do fluxo) foi também estudada dentro da segunda camada. Foi observado que perto da entrada do molde $(X<8 \mathrm{~mm})$ as fibras são orientadas perpendicularmente ao fluxo. Para distâncias entre $8 \mathrm{~mm}$ e 170 $\mathrm{mm}$, as fibras são orientadas paralelamente ao fluxo e perto do fim do molde, as fibras são orientadas perpendicularmente ao fluxo. Este comportamento pode ser visto nas Figuras 9a até 9d que mostram a orientação das fibras em função da posição dentro do molde (direção fluxo) numa profundidade de $+0.75 \mathrm{~mm}$ perto da entrada (Figura 9a e 9b) no meio do molde (Figura 9c)e perto do fim do molde (Figura 9d). As localizações das amostras (A a D) são mostradas na Figura 1.

\section{Efeito da adição de compatibilizante}

A Figura 10 mostra a morfologia de uma amostra injetada de PP/PA não compatibilizada. Pode ser ob-

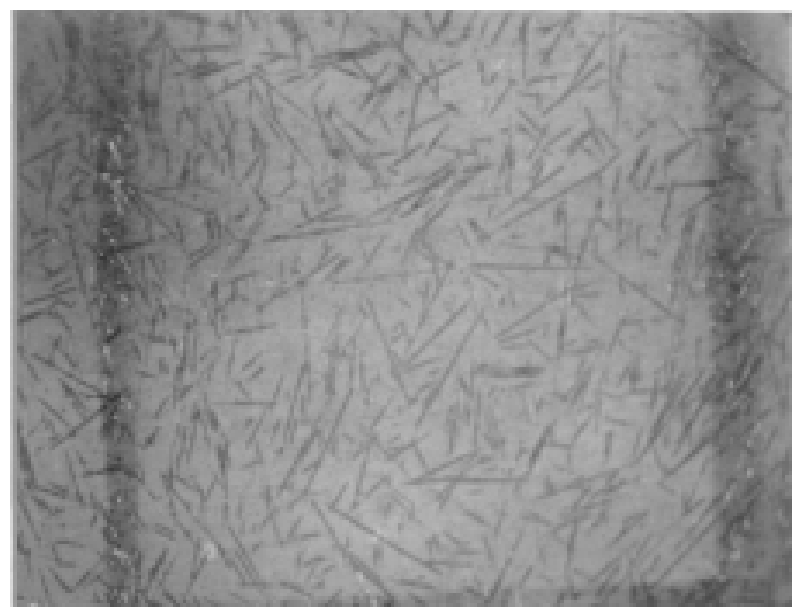

(b)

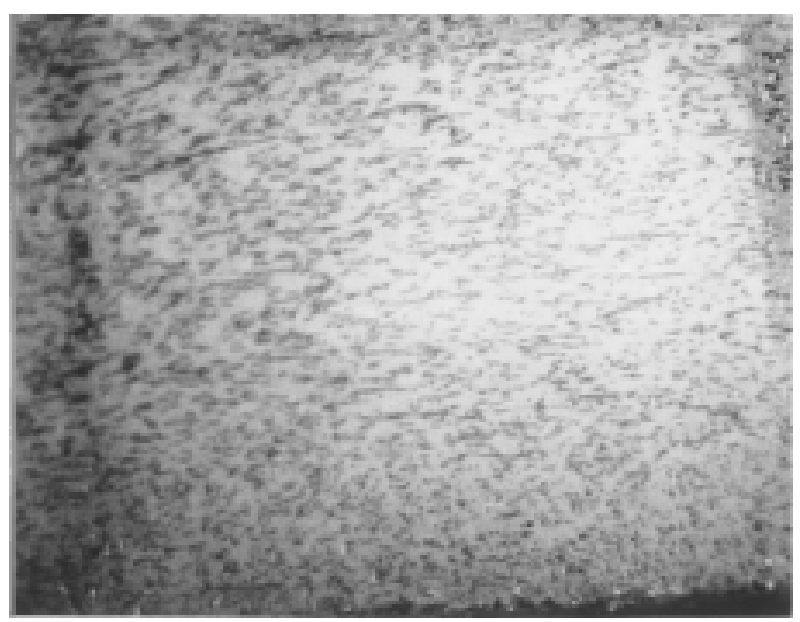

(d) 


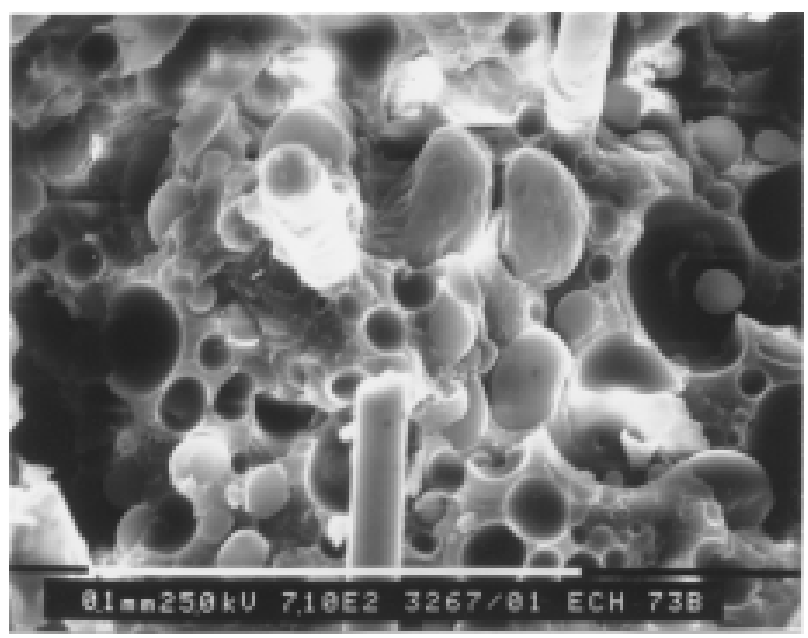

Figura 10. Morfologia de uma blenda de PP/PA (70/30) não compatibilizada sem ataque químico. Tinj $=280{ }^{\circ} \mathrm{C}$; Tmolde $=120{ }^{\circ} \mathrm{C}$; Vinj $=55,2 \mathrm{~mm} / \mathrm{s}$

servado que não existe adesão entre a fase dispersa e a fase matriz; o tamanho da fase dispersa é de $10 \mu \mathrm{m}$ a $20 \mu \mathrm{m}$. As Figuras 11a e 11b mostram a morfologia de uma amostra compatibilizada de PP/PA obtida nas mesmas condições de processamento. A Figura 11a corresponde à observação morfológica antes de qualquer tratamento químico. É bastante difícil distinguir a fase dispersa de PP devido à boa adesão entre as duas fases. A Figura 11b mostra a morfologia da mesma amostra após ataque químico. Pode ser observado que o tamanho da fase dispersa na blenda compatibilizada diminui para um tamanho médio de $2-3 \mu \mathrm{m}$.

As Figuras 12a a $12 \mathrm{~d}$ mostram as morfologias de amostras de PP/PA injetadas compatibilizadas (12a e 12 b) ou não (12c e 12d) na direção do fluxo (Figuras 12a e 12c) e na direção perpendicular ao fluxo (12b e 12d). A fase dispersa (PP) das amos-

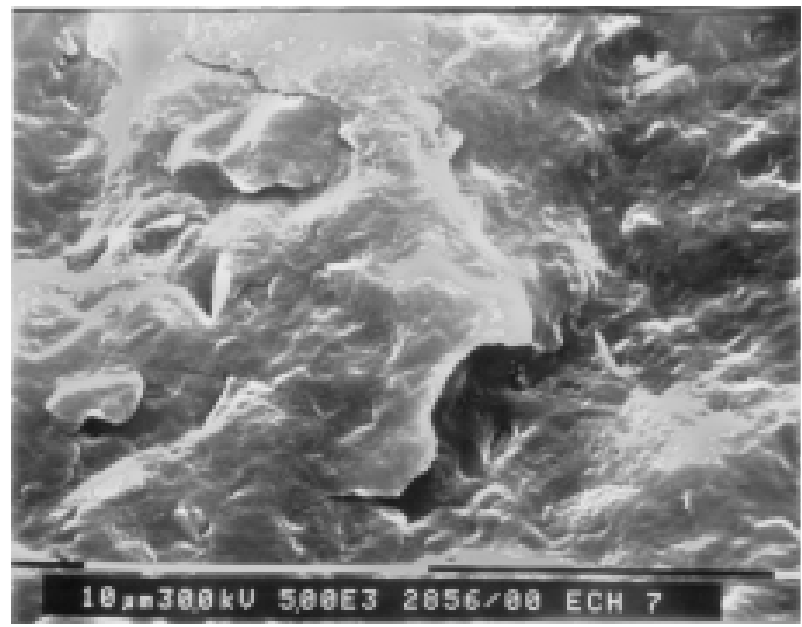

a) Antes do ataque químico.

Figura 11. Morfologia de uma blenda de PP/PA (70/30) compatibilizada tras compatibilizadas foi retirada por ataque químico. Pode ser vista a pobre adesão que existe entre o PP e o PA nas Figuras 12c e 12d quando as blendas não são compatibilizadas. Comparando-se as figuras $12 \mathrm{a}$ e $12 \mathrm{c}$ e as figuras $12 \mathrm{~b}$ e $12 \mathrm{~d}$ pode ser observado o efeito da compatibilização no tamanho da fase dispersa. O tamanho da fase dispersa passa de 10-20 $\mu \mathrm{m}$ para as blendas não compatibilizadas para até $1-2 \mu \mathrm{m}$ no caso das blendas compatibilizadas. Comparando-se as Figuras 12c e 12d pode ser visto que a fase dispersa é mais "esticada" na direção paralela ao fluxo no caso das blendas não compatibilizadas. Este efeito não é observado para as blendas compatibilizadas (Figura 12a e 12b) mostrando o papel do compatibilizante como estabilizador de morfologia. Este papel de estabilizador de morfologia já tem sido observado por outros pesquisadores no caso da blenda de poliestireno com polietileno de alta densidade ${ }^{[19]}$.

\section{Efeito da velocidade de injeção}

As Figuras 13a e 13b mostram as morfologias de duas amostras de PP/PA compatibilizadas para duas velocidades de injeção, respectivamente $18,3 \mathrm{~mm} / \mathrm{s}$ e $55,2 \mathrm{~mm} / \mathrm{s}$. Pode ser visto que o tamanho da fase dispersa é menor para velocidades de injeção maiores.

\section{Comportamento Mecânico}

A Tabela 3 mostra a resistência à ruptura, o módulo de elasticidade e a resistência ao impacto das blendas compatibilizadas ou não, para diferentes parâmetros de injeção.

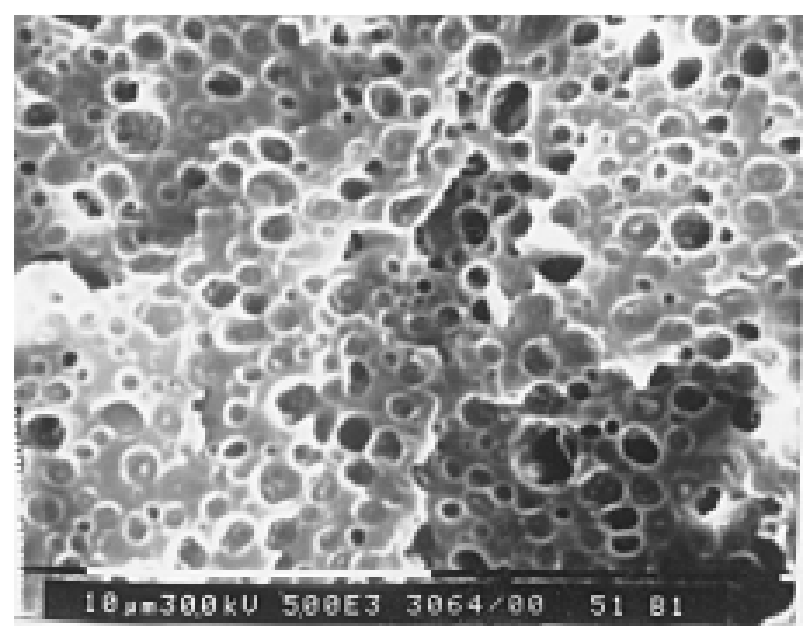

b) Após ataque químico. Tinj $=280^{\circ} \mathrm{C} ;$ Tmolde $=120^{\circ} \mathrm{C} ; \mathrm{Vinj}=55,2 \mathrm{~mm} / \mathrm{sb}$ ) 


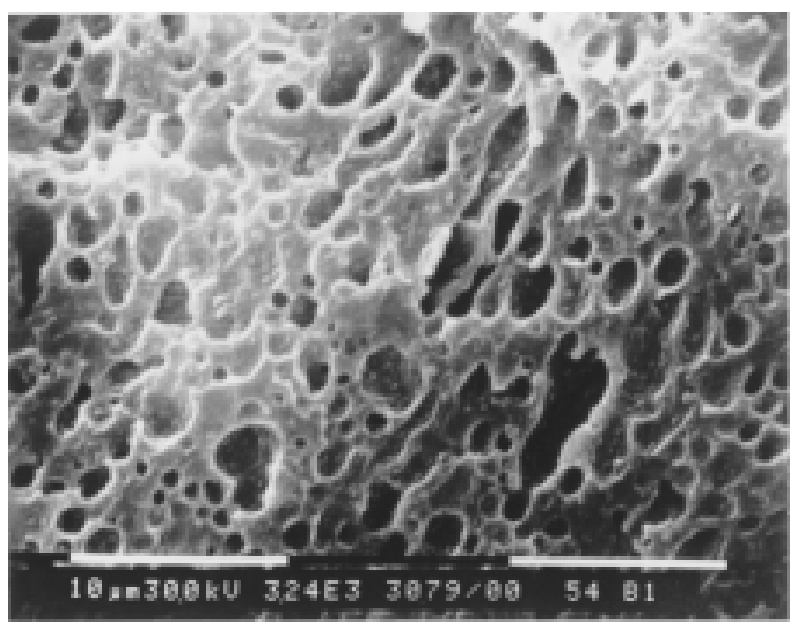

(a) Blenda compatibilizada na direção do fluxo

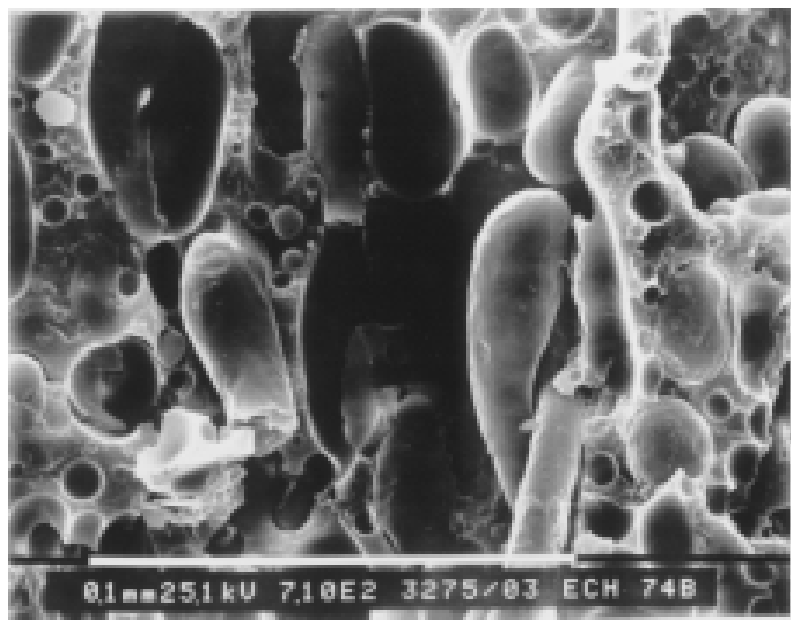

(c) Blenda não compatibilizada na direção do fluxo

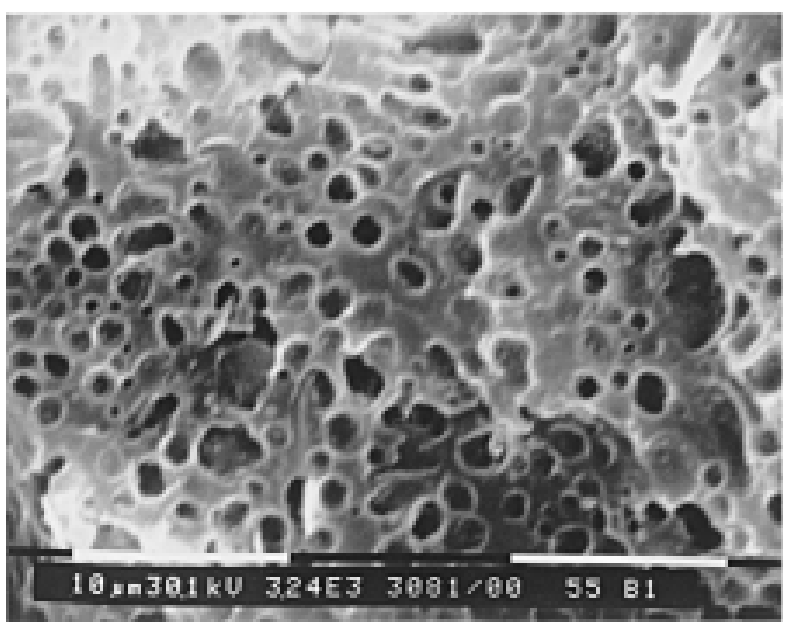

(b) Blenda compatibilizada na direção perpendicular ao fluxo

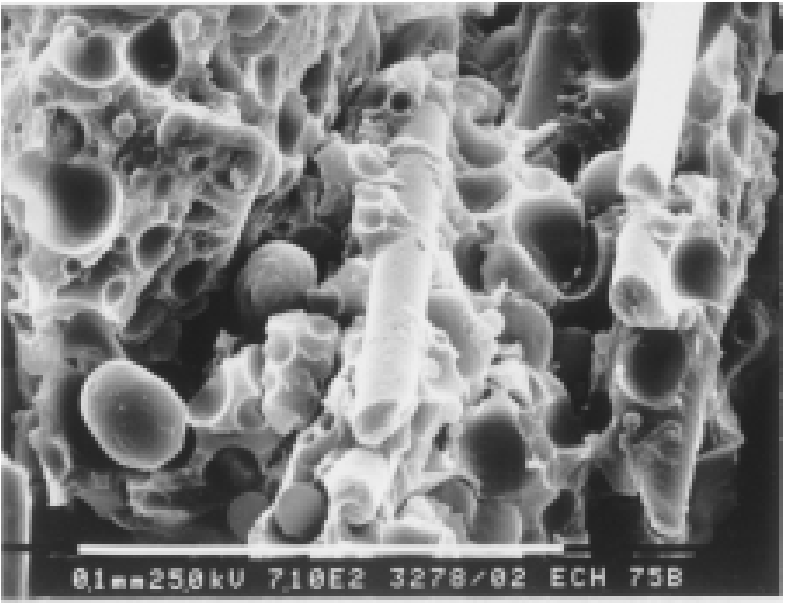

(d) Blenda não compatibilizada na direção perpendicular ao fluxo

Figura 12. Morfologia de blendas de PP/PA (70/30) injetadas $\left(T\right.$ injeção $=280^{\circ} \mathrm{C}$; Tmolde $=120^{\circ} \mathrm{C}, \mathrm{V}$ injeção $\left.=36,6 \mathrm{~mm} / \mathrm{s}\right)$

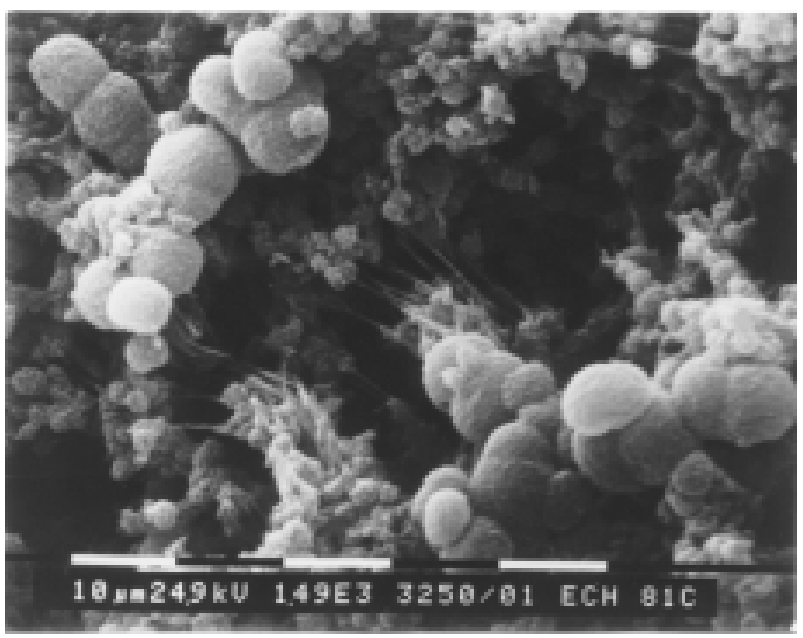

(a) $18,3 \mathrm{~mm} / \mathrm{s}$

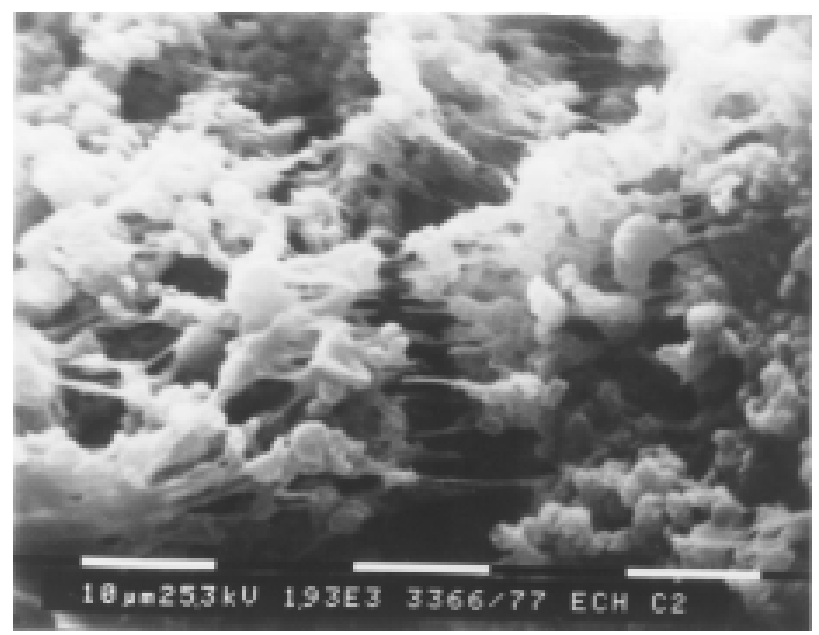

(b) $55,2 \mathrm{~mm} / \mathrm{s}$

Figura 13. Morfologia de amostras de $\mathrm{PP}_{\mathrm{g}} / \mathrm{PA}(70 / 30)$ em função da velocidade de injeção $\left(\mathrm{T}\right.$ injeção $=280^{\circ} \mathrm{C} ;$ Tmolde $\left.=120^{\circ} \mathrm{s} \mathrm{C}\right)$

Pode ser concluído a partir dos resultados apresentados na Tabela 3 que, para blendas compatibilizadas ou não, a resistência à ruptura, o módulo de elasticidade e a resistência ao impacto aumentam quando a velocidade de injeção passa de 18,3 para 55,2 $\mathrm{mm} / \mathrm{s}$. Os resultados de resistência à tração podem ser 
Tabela 3. Comportamento das blendas em função dos parâmetros de injeção

\begin{tabular}{lcccccc}
\hline & Vinj (mm/s) & $\begin{array}{c}\text { T de injeção } \\
\left({ }^{\circ} \mathbf{C}\right)\end{array}$ & $\begin{array}{c}\text { T do molde } \\
\left({ }^{\circ} \mathbf{C}\right)\end{array}$ & $\begin{array}{c}\text { Módulo de } \\
\text { Elasticidade } \\
(\mathbf{G p a})\end{array}$ & $\begin{array}{c}\text { Resistência à } \\
\text { ruptura } \\
(\mathbf{M P a})\end{array}$ & $\begin{array}{c}\text { Resistência } \\
\text { ao impacto Charpy } \\
\left(\mathbf{K J} / \mathbf{m}^{2}\right)\end{array}$ \\
\hline $\mathrm{PP} / \mathrm{PA}$ & 18,3 & 280 & 60 & 9,6 & 141 & 22,1 \\
$\mathrm{PP} / \mathrm{PA}$ & 55,2 & 280 & 60 & 9,9 & 148 & 23,5 \\
$\mathrm{PPg} / \mathrm{PA}$ & 18,3 & 280 & 60 & 9,8 & 142 & 33 \\
$\mathrm{PPg} / \mathrm{PA}$ & 55,2 & 280 & 60 & 10,1 & 144 & 30,2 \\
$\mathrm{PP} / \mathrm{PA}$ & 18,3 & 280 & 120 & 9,8 & 143 & 19 \\
$\mathrm{PPg} / \mathrm{PA}$ & 18,3 & 280 & 120 & 10,7 & - & 23,4 \\
$\mathrm{PP} / \mathrm{PA}$ & 55,2 & 280 & 120 & - & - & 17 \\
$\mathrm{PPg} / \mathrm{PA}$ & 55,2 & 280 & 120 & - & - & 29,3 \\
\hline
\end{tabular}

explicados pela orientação das fibras ser mais importante para velocidades de injeção mais altas. $\mathrm{O}$ aumento na resistência ao impacto pode ser explicado pela morfologia mais fina obtida para velocidades de injeção mais altas como foi visto na Figura 13. Quando a temperatura do molde varia de $60^{\circ} \mathrm{C}$ para $120^{\circ} \mathrm{C}$ pode ser observado que a resistência à tração e o módulo de elasticidade aumentam enquanto a resistência ao impacto diminui para todas as blendas. Pode ser visto que para as blendas compatibilizadas a diminuição na resistência ao impacto é menor do que para as blendas não compatibilizadas. Este comportamento pode ser explicado pela coalescência da fase dispersa ser mais importante para temperaturas mais elevadas para blendas não compatibilizadas e a coalescência menor no caso das blendas compatibilizadas.

\section{Conclusões}

Neste trabalho foi estudada a injeção de blendas de polipropileno com poliamidas aromáticas reforçadas com fibras de vidro e em particular a orientação das fibras em função da posição dentro do molde de injeção, o efeito da adição de compatibilizante e da composição no comportamento reológico e morfológico bem como o efeito dos parâmetros de injeção nas propriedades mecânicas das blendas.

Foi mostrado que, utilizando-se um molde retangular especialmente projetado, a orientação das fibras varia em função da espessura e da posição dentro do molde. Foram observadas três camadas distintas na direção perpendicular ao fluxo: a)uma camada chamada de "pele", em contato direto com o molde onde as fibras são orientadas aleatoriamente; b) uma segunda camada onde as fibras estão orientadas na direção do fluxo e c) uma camada central com fibras orientadas perpendicular ao fluxo.
O compatibilizante melhora a adesão entre as fases, tem um papel de agente emulsificante e também estabiliza as morfologias das amostras injetadas.

Utilizando-se um reômetro especial "in line", localizado entre a bucha de injeção e o molde de injeção na máquina de injeção, foi possível determinar a viscosidade e a primeira diferença de tensões normais em função da composição da blenda diretamente no fluxo. Usando este reômetro é possível caracterizar o comportamento reológico do polímero dentro da injetora para taxas de cisalhamento altas, e estudar a estabilidade da blenda submetida a um tal tratamento termomecânico.

As propriedades mecânicas (resistência à tração e ao impacto) da blenda foram medidas em função da velocidade de injeção e da temperatura do molde; elas melhoraram quando a velocidade de injeção passou de 18,3 para 55,2 mm/s. Quando a temperatura do molde aumenta, a resistência à tração e o módulo de elasticidade aumentam, enquanto a resistência ao impacto diminui para todas as blendas estudadas. Este decréscimo foi menor para as blendas não compatibilizadas, sendo explicado pela ausência de coalescência da fase dispersa no caso das blendas compatibilizadas.

\section{Agradecimentos}

Os autores gostariam de agradecer à FAPESP pelo auxilio à vinda de pesquisador visitante, Processo $\mathrm{N}^{\circ}$ 97/05935-3.

\section{Referências Bibliográficas}

1. Utracki, L.A - Polymer Engineering and Science, 32, 24, p.1801 (1992)

2. Juliano, P.C. - Polyblends 95, p 8 (1995) 
3. Haddout, A; El Ghorba, M; Villoutreix, G. - Proc $2^{\circ}$ Congrés de Mécanique (Societé Marocaine des Sciences Mécaniques, Casablanca, Maroc), 2, 124 (1995).

4. Haddout, A.; Villoutreix, G. - Brevet D'invention N/ Ref 233 498D. 14028DL Francês (1992).

5. Haddout, A.; Villoutreix, J. - Intern J. Composites, 25 (2), 147 (1994).

6. Gioan, P. - Actual. Chem. March, p.97 (1988).

7. U.S. Patente 4404312, Toyo Boseki, (1983).

8. Ide F.; Hasegawa A. - J. Appl. Polym. Sci., 18, p. 963 (1974).

9. Haddout, A.; Elghorba, M.; Villoutreix, G. - Anais do Congrés de Mécanique, Casablanca, Maroc, 2, p. 124-130 (1995).

10. D’Orazio, L.; Mancarella, C.; Martuscelli, L.; Casale, A.; Filipi, A.; Peroni, J. - J. Mater Sci., 21, p. 989 (1986).

11. D’Orazio, L.; Mancarella, C.; Martuscelli, L.; Casale, A.; Filipi, A.; Peroni, J. - J. Mater Sci., 22, p. 429 (1987).
12. Ghiam, F.; White, J.L. - Polym Eng. and Sci., 31, p. 76 (1991).

13. Karger-Kocsis - Polym. Eng. Sci.., 27, p. 241 (1989).

14. Walling, N. - Tese de Mestrado, Departamento de Engenharia Química, Universidade McGill, Montreal, Canada (1995).

15. Villoutreix, G.; Haddout, A.; Villoutreix , J. - Proc. Intern, Congress ABM, São Paulo, Brasil, 9, p. 97 (1994).

16. Carmier, J.; Villoutreix, G.; Berlot, R. - Matériaux et Tehcniques, p.25-30 (1990).

17. Kamal, M.R.; Tan, V. - Polym. Eng. Sci.., 19, p.558 (1979).

18. Bureau, M.N.; El Kadi, H.; Denault, J.; Dickson, J.I. - Polym, Eng. Sci. 37, n.2, p. 377-390 (1997).

19. Cigana,P.; Favis, B.D.; Jerome, R. - J. Polym. Sci. (Part B), Polymer Physics, 34, p. 1691-1700 (1997).

Recebido : 23/03/98

Aprovado : 30/10/98 